\title{
Attitudes towards End-of-Life Care in Italy: the Need for Education
}

\author{
Paolo Mazzola ${ }^{1,2}$ \\ ${ }^{1}$ Department of Health Sciences, University of Milano-Bicocca, and Geriatric Clinic, San Gerardo University Hospital, Italy \\ ${ }^{2}$ NeuroMI - Milan Center for Neuroscience, Clinical Neurosciences research area, 20126 Milano MI, Italy
}

\begin{abstract}
Received: April 29, 2015; Accepted: September 01, 2015; Published: September 10, 2015
*Corresponding author: Paolo Mazzola, Geriatric Clinic, Floor 7 C, via Pergolesi 33, 20900 Monza MB, Italy, Tel: +39 039-233-3475; Fax: +39 039-233-2220, E-mail: paolo.mazzola@unimib.it
\end{abstract}

\begin{abstract}
End-of-Life (EOL) care is directed towards patients with an advanced stage of a pathological condition, which has been deemed incurable. The concept should not be confused with 'palliative care', which is directed to alleviate an individual's suffering regardless the stage of a disease, even in concomitance with curative treatment.

Italy represents a unique scenario, where a strong cultural and Catholic background along with the mass-media heavily influence perceptions of EOL issues. The Oviedo Convention (1997), which granted legal accreditation to advance directives, has neither been ratified nor incorporated into the Italian legal system. In the last 15 years, a few cases particularly publicized by the media brought attention to this topic. They evidenced the related national inadequacies, from public opinion to healthcare education, as well as the absence of specific EOL legislation. This manuscript provides an overview of different perspectives on EOL issues in the Italian context

Existing problems include scarce dialogue and communication between patients, professionals, and caregivers, absence of a uniform EOL education and training, limited research, and delays in legislation. Even though important steps have been taken, all these aspects contribute to an incomplete EOL culture. The achievement of strong EOL practices requires the following changes: a cultural shift through investment of time and resources, promotion of dialogue and communication, implementation of an appropriate training program, and introducing real values to advance directives. Therefore, successful implementation of these changes will lead to a proper legislative framework.
\end{abstract}

Keywords: End-of-life; End-of-life care; Medical ethics; Italy; Terminal condition

\section{End-of-Life Care: Avoid Confusion}

The term End-of-life (EOL) care refers to the broad healthcare field that focuses on people experiencing a terminal illness, i.e. an advanced condition, which is deemed incurable [1]. EOL care should not be confused with Palliative Care (PC), which is aimed to prevent or alleviate patients' suffering, regardless of the type and stage of disease. PC includes the EOL care, but it is not limited to EOL phase [2,3]. EOL care, however, requires many decision- making competences and healthcare skills, including knowledge of PC [4]. It is intended to alleviate the patients' suffering as well as promote dialogue with the patients and their families/ caregivers, support them from a psychological and spiritual point of view, and rationalize the allocation of healthcare resources. Italy represents a unique scenario among the Western countries, where a strong cultural and Catholic background influences the EOL-related issues.

\section{Attitudes toward End-of-Life Care}

\section{The situation among healthcare professionals}

It is known that decision-making is one of the core problems for physicians who care for dying patients, because it involves different competences including ethical aspects and legal issues. To note, it is also influenced by the cultural, social and religious background, whose importance for Italy has already been mentioned. In 2006, The Italian Society of Anesthesia, Analgesia, Resuscitation and Intensive Care (SIAARTI) published a consensus document on EOL decision-making, which included the definition of persistent vegetative state and directives on artificial nutrition and hydration (ANH) [5]. This document has been constantly updated.

Recently, a debate was raised by the highly publicized case of Ms. Eluana Englaro, a 36-year-old woman who remained in a persistent vegetative state due to a car accident in 1992. After 17 years in this state, she died on February $9^{\text {th }}, 2009$, a few days after discontinuation of ANH [6]. A survey of 22,219 physicians randomly selected from all specialties, administered after the controversies generated by Ms. Englaro's death, revealed an overall unfamiliarity with advance directives and the consequent decision-making in EOL situations [7]. Despite adequate knowledge of definitions and diagnostic criteria, the experienced difficulties were primarily a reflection of Catholic influence on ethical and political debates and the absence of specific laws protecting the professionals' conduct in this arena.

More recently, a survey by Barni, et al. [8] investigated the attitude of 400 Italian oncologists toward PC for patients with 
advanced cancer. Even though it involved only oncologists affiliated with the Italian Association of Medical Oncology, and focused on cancer issues, it should be noted that they were scheduled to participate in a specific project to improve their practice and overall knowledge in EOL treatment. Interestingly, $38 \%$ of the responders reported to often discuss EOL problems with patients. Despite usually facing advanced cancer patients and the management of their symptoms, $32.8 \%$ of the interviewed referred not to deal with their EOL phase [8]. These results are in line with a previous survey carried out within the European Society of Medical Oncology (ESMO) involving 895 oncologists (from Europe, Asia and America) [9]. In particular, Italian oncologists showed similar clinical practice and attitudes toward PC compared with their international counterparts. Important differences emerged considering the direct involvement in symptoms management and the discussion about EOL care with patients and their families, both more frequent in the ESMO responders than among Italian oncologists [9]. Finally, about half of the Italian responders did not declare having received a satisfactory training in PC during his/her professional life. This result introduces the issue of $\mathrm{PC} / \mathrm{EOL}$ training.

A telephone survey of 1489 general practitioners considered their involvement in EOL home care in the context of their knowledge about PC [10]. General practitioners should play a fundamental role in the EOL care of their assisted. The investigators showed that, despite some uncertainty related to definitions and goals of $\mathrm{PC}$, Italian general practitioners are overall aware of and prone to their duties regarding home-based EOL care, including the recognized benefit of working in teams with a physician experienced in PC [10].

In 2012, Giantin et al. described EOL decisions made by 368 nurses and 171 physicians by administering them a new questionnaire. To date, strength of this research was to include a notable number of nurses, other than physicians. By exploring different geriatric settings, the investigators found that nurses were more likely to support decisions to end life than physicians (deep sedation mostly). Overall, the study reported that a majority of death events $(51.3 \%)$ was preceded by decisions supporting life-prolonging treatments, compared to only $20.8 \%$ of deaths preceded by choices of withdrawal [11]. The same research group investigated the main EOL-related topics discussed by healthcare professionals with patients affected by terminal conditions (and their relatives) in geriatric settings [12]. Again, nurses accounted for the majority of the interviewed $(\mathrm{n}=$ $454)$, followed by physicians $(n=181)$ and psychologists $(n=81)$. Despite the variety of EOL issues to address, the authors showed that physicians tend to communicate more easily with relatives than patients, while psychologists display an opposite trend. Interestingly, nurses managed the communication with patients and families in the same way. A specific training on EOL issues was reported to be helpful in improving the communications; those skills were considered as crucial for providing appropriate EOL care.

\section{Students and trainees' attitudes}

Four papers explored students' attitudes toward EOL care. The first study, from Leombruni, et al., [13] included 193 second- year Italian medical students (University of Turin), whose overall attitudes were reported as "poor", compared with findings from other international studies. A pilot cross-sectional study, from the same team of researchers, further investigated the relationship between attitudes toward EOL care and personality traits of 165 second-year medical students [14]. Researchers found positive associations of EOL care attitudes with self-directedness and cooperativeness traits, while harm avoidance was negatively associated. A paper from Lotto et al. explored the relationship between moral principles ("sanctity of life" and "free choice" principles) of 202 undergraduate students (University of Padua) and attitudes towards perception of death and EOL decision to withdraw life support. Interestingly, the authors found that moral principles in people supporting the "sanctity of life" are not necessarily related with their religious background. Moreover, Lotto and colleagues reported a more "controversial" definition of life in those supporting the "free choice" principle. Despite the different opinions and relationships about withdrawing life support ("free choice" supporters are more likely to judge the life-sustaining treatment as "appropriate"), the investigators pointed out the additional and more profound question of how to deal with different perceptions of death and life status [15]. The most recent study, again from Leombruni, et al. [16] was performed on 83 second-year nursing students (University of Turin) and showed similar results as those observed in the medical students (overall unfamiliarity with the EOL issues).

In Italy, no uniform faculty program about EOL care has been developed, and a richer education is needed beginning in undergraduate courses.

In May 2007, a reform was introduced for regulation on specialization in anesthesia and intensive care. The new curriculum included EOL communication skills and bioethical aspects among the competences required of the trainees in anesthesia and intensive care, even though their specific aims were not further described [17]. To note, these competences are widely recognized as important requirements for healthcare professionals [18]. Moreover, insufficient training represents a barrier to the development of effective EOL practices [17-19]. Since intensive care specialists are not the only professionals interacting with dying patients, it is desirable that the EOL basics would be included in the curriculum of all medical students and residents.

\section{The non-professionals' point of view}

Although cancer is only one of the possible conditions for which people are confronted with EOL issues, the Italian Survey of the Dying of Cancer (ISDOC) [20], gives perspective on the point of view of non-professional caregivers:

i. The original survey, administered to 1289 caregivers, reported that $34.6 \%$ of cancer patients died in hospital, while $57.9 \%$ at home, with wide geographical variability [21]. Without significant differences across the country, an estimated $93.5 \%$ of the interviewees reported 'home' as the preferred place of death [21]. 
A different analysis, reporting the experiences of 1158 caregivers, showed that EOL care of a terminally ill relative was a negative experience for $65 \%$ of the interviewees [22]. In $12.1 \%$ of the cases, the negative experiences were related to a perceived inadequacy in the psychological and physical support provided by the healthcare services.

A further ISDOC population-based survey focused on the caregivers of 364 patients who died in a hospital: according to their reports, $2 / 3$ of the patients did not receive basic information about the administered treatment. Caregivers experienced a lack of information from the healthcare professionals regarding the care of the patient and his or her eventual imminent death [23].

Once again, dialogue and communication in this area are perceived as inadequate. More recently, a survey focusing on PC, administered to a random sample of about 1900 adults (18-74 years old), showed an overall poor perception of this service, including communication of PC definition and goals. To note, the utilization of PC services is strongly influenced by the media, which perpetuates misperceptions directing the attitudes of patients and their families towards medical interventions rather than a quality-of-life-focused care [24]

\section{Legislation}

The Convention on Human Rights and Biomedicine (Oviedo, 1997), which granted legal accreditation to advance directives in many countries, has not been incorporated into the Italian legal system yet. Three cases, in particular, have raised the topic of advance directives from 1998 to today, all of them highly touted by the media.

i. In 1998, a man threatened the staff of an ICU with an unloaded gun, while he personally disconnected the ventilator from his wife and let her die. Indeed, he was aware of her irreparable brain condition [25].

The case of Piergiorgio Welby, a 60-year-old man who was paralyzed by severe muscular dystrophy for about 40 years. In 2006, after the worsening of his condition, he wrote a plea to the Italian president to be allowed to die [26, 27], which was not granted, and subsequently lost his legal battle for the discontinuation of mechanical ventilation. On December $23^{\text {rd }}$, 2006, following Mr. Welby's advance directives, an anesthetist chose to administer him sedatives and disconnected the ventilation machine allowing him to die [27]. After this case, a draft bill entitled "Treatment of terminally ill patients" was proposed by the Senator Giorgio Benvenuto (available at http:// www.senato.it/japp/bgt/showdoc/frame.jsp?tipodoc=Ddlpres\& leg $=15 \&$ id $=00209092 \&$ offset $=434 \&$ length $=19968 \&$ parse $=$ si\&to $\mathrm{c}=$ no $)$.

The recent case of Ms. Englaro [6] which included a long legal battle regarding her father's request of ANH discontinuation was highly publicized. In this occasion, the request was granted by the Italian Supreme Court at the end of 2008, in line with young Eluana's advance directives (as reported by her father) [6] .The majority coalition - supported by the Catholic Church-tried unsuccessfully to oppose to this decision: Eluana Englaro died before the discussion of an emergency legislative decree aimed to restore ANH support.

Prompted by these controversies, in February 2009, a second bill entitled "Provisions on the therapeutic alliance, informed consent and advanced declaration of treatment" was presented to the Italian Senate. In contrast with the definition given by the literature, the bill defined "nutrition and hydration in all forms offered by science and technology" as basic care support. The bill was widely criticized, mainly because of:

i. Its definition of ANH as basic care support, which delegitimized the advance directives oriented toward ANH discontinuation [28];

ii. Its approach defined as "reductionist"[29];

iii. The strongest value recognized was "physical survival" (instead of quality of life), which theoretically directed physicians to treat/over-treat[29];

iv. The fact that it was quickly proposed amidst the wave of controversies raised by a single case [30].

To note, the only two Italian laws currently recognizing value to advance directives are:

"Provisions relating to transplant of organs and tissues" (Legge no. 91, 1 April 1999, and G.U. no. 87, 15 April 1999, available at http://www.parlamento.it/parlam/leggi/99091l. htm).

"Support administrator" (Legge no. 6, 9 January 2004, and G.U. no. 14, 19 January 2004; http://www.camera.it/parlam/ leggi/04006l.htm), which states that an individual can define, according to his/her own preferences, the person to designate as support administrator. The role of this figure, appointed by the court, includes the legal involvement in healthcare decisionmaking (including the EOL phase) in the case of incompetence of the individual.

In the days that preceded the completion of this manuscript, a judgment of the Italian State Council declared that the 2009 decision by the Lombardy region to block the performance of ANH discontinuation of Ms. Englaro on the Lombardy territory was unlawful (Sentenza del Consiglio di Stato n. 04460 del 2/09/ 2014, available in Italian at http://www.giustizia-amministrativa. it/DocumentiGA/Consiglio\%2 0di\%20Stato/Sezione \%20 3/2009/200903000/Provvedimenti/201404460_11.XML accessed on September $\left.5^{\text {th }}, 2014\right)$. Such a declaration, which was filed on September $2^{\text {nd }}, 2014$, further underlines the inadequacy of the current legislation. Indeed, Ms. Englaro would have had the right to fulfill her advance directives in her hometown in 2009 , instead of being transferred to another region for ANH discontinuation.

\section{Overview of EOL Issues in the World}

Without the intent of describing every country, this section briefly depicts common issues related to EOL care internationally. A recent systematic review within the PRISMA project compared EOL attitudes between Italy, Spain and 
Portugal focusing on cultural aspects. Although these countries are often referred to as "Southern Europe", the existing difference does not allow portraying this geographic area as a whole. The similarities include profound within-country heterogeneity of the distribution of EOL facilities, poor dissemination of advance directives, a scarce utilization of all medical EOL decisions, and the issue of partial disclosure in Italy and Spain. Also, Catholicism and the central role of the family are considered by the literature as the main cultural aspects that influence EOL preferences and procedures in these countries. Finally, this review stressed on the risk of simplifying a Southern Europe stereotype, and enlightened that the majority of qualitative research is represented by partial studies with a very specific focus, thus not suitable to completely describe EOL care in its entirety [31]. Compared with some Northern European countries (The Netherlands, Denmark, Belgium), Italian physicians showed less familiarity with EOL decisions in a 2008 survey [32]. In particular, the authors speculate that the Netherlands is traditionally more liberal and respectful toward patients' advance directives. Moreover, the results were not significantly affected by the religious background, because Sweden (Protestant) demonstrated the lowest familiarity with EOL decision-making together with Italy (Catholic), while Belgium (prevalently Catholic) was second only to the Netherlands [32]. To date, EOL legislation mirrors the experience of physicians in every country, and some EOL procedures are more strongly affected by situational factors (e.g. acute pain and suffering) rather than the existing laws. More definitive decisions, such as euthanasia or physicianassisted suicide, are legal only in the Netherlands, Belgium, and Switzerland, under strict circumstances.

Because of the complexity and diversity of EOL care, and considering that culture is a multilayered concept that influences EOL attitudes and practices, challenging scenarios are identifiable worldwide. For example, in the United States the experience of dying is often related to the issue of fragmented care and to the frequent transitions among different care settings. Other problems, similarly to other countries, are the lack of coordination between settings, the heterogeneity of symptom management which is often reported to be inadequate -and the huge burden of the EOL phase on patients and caregivers [33]. In Canada, a pressing public priority is represented by the improvement of advance care planning in terms of both quality and quantity [34]. Similarly, there are gaps between people's preferences about EOL procedures and the care that patients actually receive [35]. Again, some authors point out that knowledge and a better training curriculum about PC and EOL care should be initiated earlier in the professionals' career [36]. In summary, more dedicated research, a better education, and an optimal resource allocation are perceived as essential issues to address in order to improve Canadian EOL care system [35].

In South America, Argentina is facing great changes initiated by a 2012 bill that authorized modifications in the law of patient's rights [37]. The 2009 law recognized the patients' right to reject medical treatments and supports, but only for those capable of making decisions. This legislation hole created debate and reinforced the necessity of considering advance directives while protecting physicians' conduct. In this changing scenario, more research is needed because EOL decisions - in particular those related with limiting therapeutic efforts in intensive care units - are very frequent in Argentina, Uruguay, and Brazil. A 2010 survey by Moritz, et al. [38] enlightened that the main obstacles to proactive decision-making oriented toward the limitation of therapeutic efforts were ethical and legal issues in these three countries, even though Argentina showed a higher proactive trend than Uruguay and Brazil. However, the results also related with gender and different professional experiences of the respondents. Another study performed in Brazilian ICUs demonstrated that EOL decisions are more difficult to make in incompetent patients, and family members are not frequently involved in the decisionmaking [39]. Overall, the authors reported also a disagreement between physicians and nurses or family members regarding withdrawal from mechanical ventilation. Again, the lack of regulations on advanced directives was cited as the main barrier to this decision, along with "paternalistic" expectations towards Brazilian physicians [40]. Despite an increasingly frequent attention towards EOL issues, Brazilian ICUs reported low rates of EOL decision making when compared with Europe and the USA [41].

On the other side of the world, the attention toward EOL care has increased in Japan. After the implementation of a public insurance for long-term care in 2000, the Japanese older people's attitudes have changed. In particular, a survey conducted in 2012 showed that individuals were more likely to wish to be notified about an incurable condition, to receive narcotics to alleviate respiratory discomfort, and less likely to prefer life-sustaining treatments than people interviewed in 1996 [42]. In China, the great technical advances in medical treatment and equipments experienced in the last three decades brought the issue of EOL care, from the social and ethical point of views. China significantly differs from Western countries, and its large population presents a wide variety of ethnicities, traditions, and cultural-religious background (Confucianism, Taoism, Buddhism, Catholicism, Islam) [43]. In particular, the traditional Chinese ethics together with the availability of life-support technologies and the absence of specific laws frequently lead to overtreatment of elderly patients with incurable conditions in their EOL phase. The older segment of Chinese population (aged $\geq 60$ years) currently accounts for more than 200 million individuals, which is more than the total of European elderly subjects. In this scenario, resources are limited and more research is needed to understand the multiple bioethical aspects, to develop corresponding guidelines, and to draft legislation concerning EOL care [43].

Finally, an interesting perspective came from Australia, where some authors suggested improving an already advanced EOL care system by switching the setting of EOL decision-making from the Palliative Care system to the acute hospital setting. In other words, the acute care specialists should effectively become the "gatekeepers" to the palliative care system, with a patientand family-centered decision-making process oriented towards quality of life in the EOL phase, and addressing the patients to the 
most appropriate setting [44]. To date, the different Australian healthcare professionals recognized EOL issues as the most challenging ethical issues that they routinely face within the acute care medical setting.

\section{Limitations}

This manuscript does not cover all the areas of EOL care, which are multiple and diverse, but rather focuses on attitudes by considering the available studies. The majority of them consider terminal cancer or persistent vegetative state, thus limiting the possibility to generalize the considerations.

\section{How to provide end-of-life education?}

EOL issues are complex, require solid and different competences, and have vast implications. The Italian scenario, despite significant improvements in the promotion of these issues, still enlightens a strong influence of mass-media and cultural-social-religious background on the professional and non-professional population. National cases involving EOL and advance directives confirmed the inadequacy of how these important healthcare aspects are perceived, regulated, and experienced. Valuable guidelines do exist, but the legislation is still delayed. The topic is considered of high importance, but the recent political maneuvers penalized research.

For all these reasons, the only way for an adequate regulation of EOL issues seems to be the establishment of an alternative culture which would embrace the entire society, where dialogue is encouraged and death is not exclusively seen as a defeat, and where education and research are promoted [45]. Figure 1 summarizes the variety of elements to be involved, from general population to higher education and the institutions. Moral principles and opinions could be different, but communication is essential to achieve an agreement that is far to be accomplished.

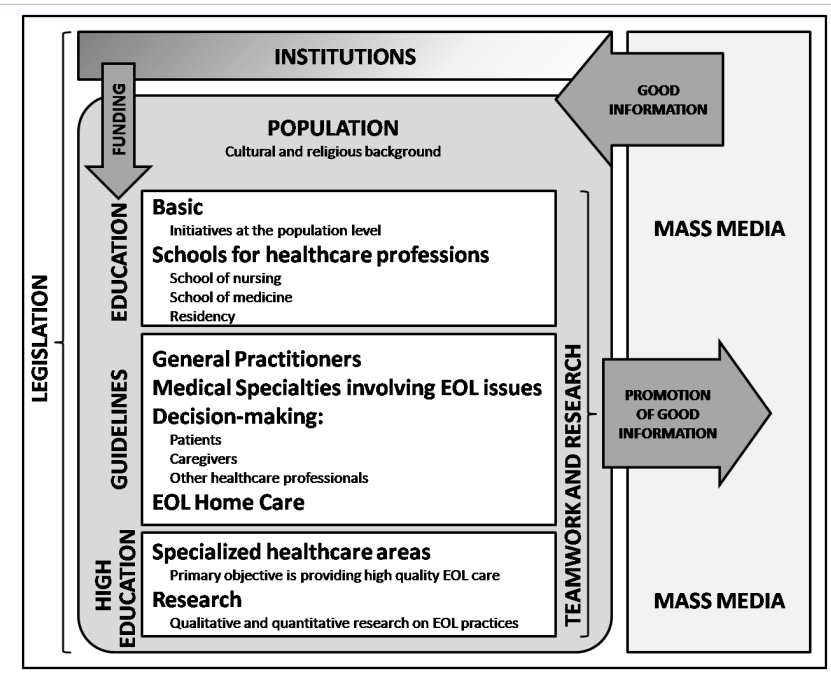

Figure 1: The scheme summarizes the elements of the society to be involved in the cultural shift. Their interconnections emphasize the need for a global focus toward EOL care issues and initiatives, which would influence and help creating an alternative culture.
This cultural shift should include the implementation of a uniform training (beginning with the undergraduate medical and nursing classes), the defense of the autonomy of physicians according to the Medical Deontological Code, the improvement of communication between doctors and patients/caregivers, and the possibility to give real value to the patients' advance directives.

Education on EOL care represents the foundation to disseminate knowledge in the general population. EOL issues pertain to many areas of medicine, including anesthesiology, internal and geriatric medicine, oncology, hematology, neurology, and even pediatric medicine and general practice. For this reason, education should begin at medical school and then continue during the postgraduate residency programs, particularly those in the medical and surgical areas.

Because of the extent, the variety, and specificity of topics, part of the lessons should provide for an "optional" participation, for example EOL issues in pediatrics or in the ICU, for future specialists in those fields. Up to now, as previously enlightened [17], the 2006 reform of regulations on anesthesia and intensive care residency program was the only one that briefly included the knowledge of "administrative procedures and the relevant managerial, bioethical, and legal implications of the discipline" in its "integrative objectives". Even though the importance of communication skills is briefly acknowledged, other core aspects of palliative and EOL care are not developed in the "educational objectives" [17].

An appropriate program should perhaps include at least three sections: basis of EOL, advanced EOL issues, and "specific aspects" of EOL. A possible scheme of the multiple topics to be covered is reported in Figure 2. Ideally, some of the topics should be also available to the general public, for example by organizing courses through the year and by implementing e-learning platforms, accessible after a simple registration process in order to monitor their dissemination efficacy.

As Doyle wrote in the introduction of his Textbook of Palliative Care in 1998, "the principles of palliative medicine apply whenever men and women suffer and die. [...] everywhere, whatever the (physicians') specialty, their culture and religious beliefs. [...] Tomorrow, the principles of palliative medicine should be the norm worldwide" [46].

Trying to realize these goals requires a huge investment of time and resources, including research funding. From that point onwards, once laid this solid foundation, a new and more appropriate bill would probably accompany the whole process and help instill greater security.

\section{Acknowledgments}

The author expresses sincere appreciation to Dr. Andrea Croccolo and to Justin S. Brathwaite, B.A., volunteer at Icahn School of Medicine at Mount Sinai Medical Center - Department of Emergency Medicine (New York), for the contribution in reviewing the English version of this manuscript. 


\section{EOL Education Program}

\begin{tabular}{|c|}
\hline $\begin{array}{l}\text { BASIS OF EOL } \\
\text { Principles of communication } \\
\text { Culture and language in communication } \\
\text { Principles of EOL and advance directives } \\
\text { Assessment of the patients at EOL } \\
\text { The variety of EOL scenarios }\end{array}$ \\
\hline $\begin{array}{l}\text { ADVANCED EOL ISSUES } \\
\text { EOL decision-making and planning } \\
\text { Symptom management and emergencies } \\
\text { Economy and resources allocation in EOL } \\
\text { Last days of life } \\
\text { EOL home and nursing home care }\end{array}$ \\
\hline $\begin{array}{l}\quad \text { SPECIFIC ASPECTS OF EOL } \\
\text { Condition-specific EOL cases } \\
\text { Field-specific EOL cases } \\
\text { Spirituality and phylosophy of EOL } \\
\text { Social support of patients at the EOL } \\
\text { Bereavement }\end{array}$ \\
\hline
\end{tabular}

Figure 1: A possible three-step program for the implementation of EOL education in Italy, from medical and nursing schools to the general public.

\section{References}

1. Wolpert CM, Menold MM, Bass MP, Qumsiyeh MB, Donnelly SL, Ravan SA, et al. Three probands with autistic disorder and isodicentric chromosome 15. Am J Med Genet. 2000;96(3):365-372.

2. World Health Organization. National cancer control programmes:policies and managerial guidelines. 2 ed.Geneva:WHO. 2002.

3. Pastrana T, Junger S, Ostgathe C, Elsner F, Radbruch L. A matter of definition--key elements identified in a discourse analysis of definitions of palliative care. Palliat Med. 2008;22(3):222-232. doi:22/3/222[pii] 10.1177/0269216308089803

4. Reid CM, Gibbins J, McCoubrie R, Forbes K. Palliative care is not same as end of life care. BMJ 2011;342:d2735.

5. Huang HQ, Zhang X, Shen YH, Su J, Liu XH, Tian JM, et al. Polyacetylenes from Bupleurum longiradiatum. J Nat Prod. 2009;72(12):2153-2157. doi: $10.1021 / \mathrm{np} 900534 \mathrm{v}$.

6. Luchetti M. Eluana Englaro, chronicle of a death foretold: ethical considerations on the recent right-to-die case in Italy. J Med Ethics. 2010;36(6):333-335. doi: jme.2009.034835 [pii] 10.1136/ jme.2009.034835

7. Solarino B, Bruno F, Frati G, Dell'erba A, Frati P. A national survey of Italian physicians' attitudes towards end-of-life decisions following the death of Eluana Englaro. Intensive Care Med. 2011:37(3);542549. doi:10.1007/s00134-011-2132-5

8. Barni S, Maltoni M, Tuveri G, Pronzato P, Cortesi E, Massidda B ,et al. Attitude of Italian medical oncologists toward palliative care for patients with advanced cancer: results of the SIO project. Support Care Cancer. 2011;19(3):381-389. doi: 10.1007/s00520-010-0831-z.

9. Cherny NI, Catane R. Attitudes of medical oncologists toward palliative care for patients with advanced and incurable cancer: report on a survery by the European Society of Medical Oncology Taskforce on Palliative and Supportive Care. Cancer. 2003;98(11):2502-2510. doi: 10.1002/cncr.11815

10. Beccaro M, Lora Aprile P, Scaccabarozzi G, Cancian M, Costantini M. Survey of Italian general practitioners: knowledge, opinions, and activities of palliative care. J Pain Symptom Manage. 2013:46(3):335-344. doi:S0885-3924(12)00503-9 [pii] 10.1016/j. jpainsymman.2012.08.020

11. Giantin V, Siviero P, Simonato M, Iasevoli M, Pengo V, Andrigo M et al. Physicians' and nurses' experiences of end-of-life decision-making in geriatric settings. Aging Clin Exp Res. 2012;24(5):537-547. doi: $10.3275 / 8588$

12. Iasevoli M, Giantin V, Voci A, Valentini E, Zurlo A, Maggi S, et al. Discussing end-of-life care issues with terminally ill patients and their relatives: comparisons among physicians, nurses and psychologists. Aging Clin Exp Res. 2012;24(3 Suppl):35-42. doi: 8712 [pii]

13. Leombruni P, Miniotti M, Bovero A, Castelli L, Torta RG. Second-year Italian medical students' attitudes toward care of the dying patient: an exploratory study. J Cancer Educ. 2012;27(4):759-763. doi: 10.1007/ s13187-012-0382-8

14. Leombruni P, Miniotti M, Torta RG. Personality and attitudes towards dying patients: an Italian pilot study among medical students. Med Teach. 2013;35(9):790. doi:10.3109/0142159X.2013.786809

15. Lotto L, Manfrinati A, Rigoni D, Rumiati R, Sartori G, Birbaumer N. Attitudes towards end-of-life decisions and the subjective concepts of consciousness: an empirical analysis. PLoS One. 2012;7(2):e31735 doi: 10.1371/journal.pone.0031735

16. Leombruni P, Miniotti, M., Bovero, A., Zizzi, F., Castelli, L., Torta, R. Attitudes towards caring for dying patients: an overview among Italian nursing students and preliminary psychometrics of the FATCOD-B scale. Journal of Nursing Education and Practice. 2014;4(3):9.. doi:10.5430/jnep.v4n3p188

17. Giannini A. Ethics and end-of-life care in the new training curriculum for ICU physicians in Italy. Intensive Care Med. 2007;33(6):10431045. doi: $10.1007 / \mathrm{s} 00134-007-0653-8$

18. Hickey M. What are the needs of families of critically ill patients? A review of the literature since 1976. Heart Lung. 1990;19(4):401-415

19. Nelson JE, Angus DC, Weissfeld LA, Puntillo KA, Danis M, Deal D, et al. End-of-life care for the critically ill: A national intensive care unit survey. Crit Care Med. 2006;34(10):2547-2553. doi: 10.1097/01. CCM.0000239233.63425.1D

20. Costantini M, Beccaro M, Merlo F. The last three months of life of Italian cancer patients. Methods, sample characteristics and response rate of the Italian Survey of the Dying of Cancer (ISDOC). Palliat Med. 2005; 19(8):628-638

21. Beccaro M, Costantini M, Giorgi Rossi P, Miccinesi G, Grimaldi M, Bruzzi P. Actual and preferred place of death of cancer patients. Results from the Italian survey of the dying of cancer (ISDOC). J Epidemiol Community Health. 2006;60(5):412-416. doi: 10.1136/ jech.2005.043646

22. Morasso G, Costantini M, Di Leo S, et al. End-of-life care in Italy: personal experience of family caregivers. A content analysis of open questions from the Italian Survey of the Dying of Cancer (ISDOC). 
Psychooncology. 2008;17(11):1073-1080 doi: 10.1002/pon.1332

23. Beccaro M, Caraceni A, Costantini M. End-of-life care in Italian hospitals: quality of and satisfaction with care from the caregivers' point of view--results from the Italian Survey of the Dying of Cancer. J Pain Symptom Manage. 2010;39(6):1003-1015. doi: S08853924(10)00244-7 [pii] 10.1016/j.jpainsymman.2009.11.317

24. Benini F, Fabris M, Pace DS, Vernò V, Negro V, De Conno F, et al Awareness, understanding and attitudes of Italians regarding palliative care. Ann Ist Super Sanita. 2011;47(3):253-259. doi: DOI: 10.4415/ANN_11_03_03

25. Maggiore SM, Antonelli M. Euthanasia, therapeutic obstinacy or something else? An Italian case. Intensive Care Med. 2005;31(7):997998. doi: 10.1007/s00134-005-2645-x

26. Turone F. Italy debates end of life decisions. BMJ. 2006;333(7571):719 doi:333/7571/719-a [pii]10.1136/bmj.333.7571,719-a

27. Bock M, Ciarrocchi V, Wiedermann CJ. Case involving end-of-life decision issues in Italy. Intensive Care Med. 2007;33(6):1041-1042. doi:10.1007/s00134-007-0632-0

28. Gristina GR, Martin E, Ranieri VM. Regulation of advance directives in Italy: a bad law in the making. Intensive Care Med. 2012:38(11):18971900. doi:10.1007/s00134-012-2656-3

29. Zamperetti N, Bellomo R, Zappin F, Ronco C, Piccinni P. Regulation of advance directives in Italy: further considerations. Intensive Care Med. 2013;39(1):157-158. doi:10.1007/s00134-012-2710-1

30. Paterlini M. Italy urged to give end-of-life bill more time for debate. Lancet . 2009;373(9673):1413

31. Menaca A, Evans N, Andrew EV, Toscani F, Finetti S, Gómez-Batiste $\mathrm{X}$, et al. End-of-life care across Southern Europe: a critical review of cultural similarities and differences between Italy, Spain and Portugal. Crit Rev Oncol Hematol. 2012;82(3):387-401. doi:10.1016/j. critrevonc.2011.06.002.

32. Lofmark R, Nilstun $T$, Cartwright $C$, Fischer $S$, van der Heide $A$ et al. Physicians' experiences with end-of-life decision-making: survey in 6 European countries and Australia. BMC Med. 2008;6:4 doi:10.1186/1741-7015-6-4

33. Dying in America: improving quality and honoring individual preferences near the end of life. Mil Med.2015;180(4):365-367. doi:10.7205/MILMED-D-15-00005
34. Heyland DK, Barwich D, Pichora D, Dodek P, Lamontagne F, You JJ, et al. Failure to engage hospitalized elderly patients and their families in advance care planning. JAMA Intern Med. 2013;173(9):778-787 doi:10.1001/jamainternmed. 2013;180

35. Cook D, Rocker G, Heyland D. Enhancing the quality of end-of-life care in Canada. CMAJ. 2013;185(16):1383-1384. doi:10.1503/ cmaj.130716

36. Heyland DK, Cook DJ, Rocker GM, Dodek PM, Kutsogiannis DJ, Skrobik Y,et al. Defining priorities for improving end-of-life care in Canada. CMAJ. 2010;182(16):E747-752. doi: 10.1503/cmaj.100131

37. Fuertes N. End-of-life decisions in Argentina. Lancet. 2012;379(9811):105. doi: 10.1016/S0140-6736(12)60042-7

38. Moritz RD, Deicas A, Rossini JP, Silva NB, Lago PM, Machado FO. Perceptions about end of life treatment in Argentina, Brazil and Uruguay intensive care units. Rev Bras Ter Intensiva. 2010;22(2):125132. doi:S0103-507X2010000200005

39. Fumis RR, Deheinzelin D. Respiratory support withdrawal in intensive care units: families, physicians and nurses views on two hypothetical clinical scenarios. Crit Care. 2010;14(6):R235 doi:10.1186/cc9390

40. Soares M. End of life care in Brazil: the long and winding road. Crit Care. 2011;15(1):110. doi:10.1186/cc9962

41. Soares M, Terzi RG, Piva JP. End-of-life care in Brazil. Intensive Care Med. 2007;33(6):1014-1017. doi:10.1007/s00134-007-0623-1

42. Shimada C, Hirayama R, Nakazato K, Arai K, Ishizaki T, Aita K, Shimizu $\mathrm{T}$,et al. What has become more acceptable? Continuity and changes in older adults' attitudes toward end-of-life care in Japan. Geriatr Gerontol Int. 2015;15(7):927-928. doi:10.1111/ggi.12475

43. Li LB. Clinical review: Ethics and end-of-life care for critically ill patients in China. Crit Care. 2013;17(6):244. doi:10.1186/cc13140

44. McGrath P, Henderson D. Resolving end-of-life ethical concerns: important palliative care practice development issues for acute medicine in Australia. Am J Hosp Palliat Care. 2008;25(3):215-222. doi:10.1177/1049909108315514.

45. Mazzola P. End-of-life dialogue, end-of-life education. BMJ Support Palliat Care. 2014;4:2. doi:10.1136/bmjspcare-2014-000707

46. Doyle D, Hanks G, MacDonald N. Oxford Textbook of Palliative Medicine. Oxford, England: Oxford University Press;1998:3-8 